\title{
Strong variation of dielectric response and optical properties of lithium under pressure
}

\author{
V. M. Silkin, ${ }^{1}$ A. Rodriguez-Prieto, ${ }^{1,2}$ A. Bergara, ${ }^{1,2,3}$ E. V. Chulkov, ${ }^{1,3,4}$ and P. M. Echenique ${ }^{1,3,4}$ \\ ${ }^{1}$ Donostia International Physics Center (DIPC), Manuel de Lardizabal Pasealekua, E-20018 Donostia, Basque Country, Spain \\ ${ }^{2}$ Materia Kondentsatuaren Fisika Saila, Zientzi Fakultatea, Euskal Herriko Unibertsitatea, 644 Posta kutxatila, E-48080 Bilbo, \\ Basque Country, Spain \\ ${ }^{3}$ Centro Mixto CSIC-UPV/EHU, 1072 Posta kutxatila, E-20080 Donostia, Basque Country, Spain \\ ${ }^{4}$ Materialen Fisika Saila, Kimika Fakultatea, Euskal Herriko Unibertsitatea, 1072 Posta kutxatila, E-20080 Donostia, \\ Basque Country, Spain
}

(Received 21 March 2007; published 10 May 2007)

\begin{abstract}
Strong modification of bonding and electronic properties in lithium under pressure becomes the origin of its striking departure from the classical nearly-free-electron-like model. In this Brief Report, we predict the existence of a low-energy long-lived interband electronic collective mode arising with the bcc to fcc structural transition in lithium under pressure, which is expected to induce an abrupt plasma edge in the experimentally observable reflectivity and also affect electronic correlations at low energies.
\end{abstract}

DOI: $10.1103 /$ PhysRevB.75.172102

PACS number(s): 71.20.Dg, 71.45.Gm, 78.40.Kc

Recent theoretical and experimental high-pressure studies of lithium have yielded surprising and intriguing results. Lithium undergoes pressure induced structural transitions from highly compact to low-coordinated complex structures. ${ }^{1-5}$ Although at normal conditions lithium adopts a bcc structure and at around $8 \mathrm{GPa}$ the compact fcc phase starts to be favored, recent $\mathrm{x}$-ray analysis ${ }^{3}$ revealed that lithium also undergoes a fcc to a rhombohedral $h R 1$ transition at $39 \mathrm{GPa}$ and a $h R 1$ to $c I 16$ (with 16 atoms per unit cell) at $41 \mathrm{GPa}$. On the other hand, despite the increasing electric resistivity in lithium with pressure, ${ }^{6,7}$ it superconducts at around $15 \mathrm{~K}$ when the applied pressure rises to $30 \mathrm{GPa} .{ }^{8-11}$ This enhanced $T_{c}$ in lithium under pressure is even more remarkable considering that superconductivity experiments at ambient pressure have failed. ${ }^{12}$ Such an unexpected behavior is not unique of lithium, but has also been predicted and recently observed both in heavier alkalies ${ }^{13}$ and other elements. ${ }^{14}$ Additionally, correlated with the appearance of low-symmetry solid phases, sodium presents an almost room melting temperature under pressure, ${ }^{15}$ and similar or even more drastic behavior might be expected in the melting curve of lithium. ${ }^{16}$

The physical origin of all these anomalies basically relies on the profound change of bonding and electronic properties with pressure. The increasing orbital $s-p$ mixing and the associated electronic localization in lithium under pressure distort its Fermi surface, ${ }^{17}$ which shows an extended nesting that induces a phonon softening. This softening, besides preluding the observed transition to complex structures, ${ }^{18}$ also enhances the electron-phonon coupling responsible for superconductivity. ${ }^{19}$ This striking departure from the nearlyfree-electron-like model under pressure is expected to induce other yet unexplored anomalies. Here, we present an $a b$ initio theoretical analysis of pressure induced modification of the low-momentum dynamic electronic dielectric response in lithium. According to our calculations, the bcc to fcc structural transition gives rise to (besides the conventional freeelectron-like plasmon) an additional new collective mode with a lower energy. This new plasmon appears to be undamped because it cannot decay via any intrinsic simple electronic scattering mechanism. In the pressure range from
10 to $40 \mathrm{GPa}$, the absorption power gradually redistributes from the conventional plasmon to this novel feature, which might also have a profound effect on electronic correlations at low energies, as they are affected by screening of collective modes. ${ }^{20}$

In time-dependent density-functional theory, ${ }^{22}$ the linear density-response function $\chi$ is given by the following integral equation:

$$
\chi=\chi^{0}+\chi^{0}\left(V+K^{x c}\right) \chi,
$$

where $\chi^{0}\left(\mathbf{r}, \mathbf{r}^{\prime}, \omega\right)$ is the density response function of the noninteracting electron system, $V\left(\mathbf{r}-\mathbf{r}^{\prime}\right)$ is the Coulomb interaction, and $K^{x c}\left(\mathbf{r}, \mathbf{r}^{\prime}, \omega\right)$ accounts for dynamical exchangecorrelation effects. As here we are interested in the analysis of collective modes at low momentum, where exchange and correlation effects can be neglected, we set $K^{x c}=0$. For a periodic crystal, the Fourier transform of $\chi^{0}$ has the following form:

$$
\begin{aligned}
\chi_{\mathbf{G}, \mathbf{G}^{\prime}}^{0}(\mathbf{q}, \omega)= & \frac{1}{\Omega} \sum_{\mathbf{k}}^{B Z} \sum_{n, n^{\prime}} \frac{f_{n \mathbf{k}}-f_{n^{\prime} \mathbf{k}+\mathbf{q}}}{\varepsilon_{n \mathbf{k}}-\varepsilon_{n^{\prime} \mathbf{k}+\mathbf{q}}+(\omega+i \eta)} \\
& \times\left\langle\psi_{n \mathbf{k}}\left|e^{-i(\mathbf{q}+\mathbf{G}) \cdot \mathbf{r}}\right| \psi_{n^{\prime} \mathbf{k}+\mathbf{q}}\right\rangle\left\langle\psi_{n^{\prime} \mathbf{k}+\mathbf{q}}\left|e^{i\left(\mathbf{q}+\mathbf{G}^{\prime}\right) \cdot \mathbf{r}}\right| \psi_{n \mathbf{k}}\right\rangle .
\end{aligned}
$$

Here, $\mathbf{G}$ and $\mathbf{G}^{\prime}$ are the reciprocal-lattice vectors, the wave vectors $\mathbf{k}$ are in the first Brillouin zone $(\mathrm{BZ}), f_{n \mathbf{k}}$ represents the Fermi-Dirac distribution function, $\eta$ is a positive infinitesimal, and $\Omega$ is the normalization volume. The oneelectron energies $\varepsilon_{n \mathbf{k}}$ and wave functions $\psi_{n \mathbf{k}}$ represent the self-consistent electronic structure calculated within the local-density approximation to the density-functional theory with the lithium electron-ion interaction described by a norm-conserving pseudopotential. ${ }^{21}$ In the expansion of $\chi^{0}$ and $\chi, 20$ reciprocal vectors $\mathbf{G}$ have been included. Hence, local-crystal-field effects are included through the inversion of matrix representation of Eq. (1). ${ }^{23}$

In bcc lithium at ambient pressure, the only existing collective mode at low momentum is the conventional free- 


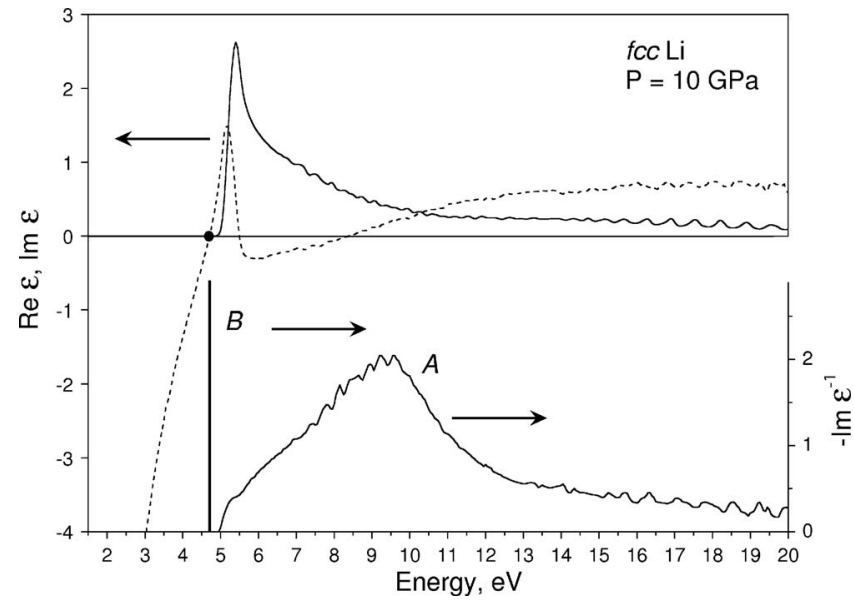

FIG. 1. (Upper panel) The calculated dielectric function $\epsilon$ for fcc lithium at $P=10 \mathrm{GPa}$. Solid (dashed) line shows imaginary (real) part of $\epsilon$. Calculations are for a small momentum $\mathbf{q}=1 / 24(2 \pi / a, 0,0)$. (Lower panel) Corresponding loss function, $-\operatorname{Im} \epsilon^{-1}$.

electron-like plasmon. Also, a weak zone-boundary plasmon has been predicted to exist at higher momenta; however, it is always strongly damped by electron-hole excitations. ${ }^{24}$ Figure 1 shows the calculated low-momentum dielectric function, $\epsilon(\mathbf{q} \rightarrow 0, \omega)$, and the corresponding loss function, -Im $\epsilon^{-1}$, for fcc lithium at $P=10 \mathrm{GPa}$, close to the bcc to fcc structural transition. Re $\epsilon$ goes to zero at three different energies in the 4-10 eV range, leading to the presence of two collective charge excitations. One of them (labeled by $A$ ) is the conventional free-electron-like plasmon, determined by the average valence electron density, which presents a wide peak at $\sim 9 \mathrm{eV}$ in the loss function. This plasmon shows a width of $2.3 \mathrm{eV}$ and is similar to the one observed for bcc lithium at equilibrium. Interestingly, another feature (labeled by $B$ ) is observed at $4.7 \mathrm{eV}$, which becomes remarkably different. At this energy, both the real and imaginary parts of $\epsilon$ go to zero and the ideal condition, $\epsilon=0$, for the existence of a well defined (undamped) plasmon is verified. Therefore, as this plasmon does not suffer from any scattering due to electron-hole excitations, it has a very long lifetime, and at $4.7 \mathrm{eV}$ the loss function shows a $\delta$-function peak, shown by a vertical line in Fig. 1. The physical origin of this striking feature is associated with the sharp increase of $\operatorname{Im} \epsilon$ at $E_{0}$ $=5.1 \mathrm{eV}$, which corresponds to the threshold energy for the optical $(\mathbf{q} \rightarrow 0)$ interband transitions around $L$, shown by gray arrows in Fig. 2, connecting electronic states with $\Delta l$ $=1$ as required for optical transitions. ${ }^{25}$ Pressure induced anisotropic flattening of bands ${ }^{1,2}$ drops electronic states near $L$ below the Fermi energy and it is precisely the presence of almost flat regions in both occupied (mainly of $p$ symmetry) and unoccupied (mainly of $s$ symmetry) states around $L$ which leads to the observed steep increase in $\operatorname{Im} \epsilon$ at $E_{0}$. Interestingly, when low-momentum dispersion of this novel feature is considered, the almost planar geometry of energy bands around $L$ makes it undamped in any direction.

In order to analyze the evolution of both plasmons under pressure, we have also performed similar calculations at $P$ $=20,30$, and $40 \mathrm{GPa}$. Figures 3 and 4 display the calculated

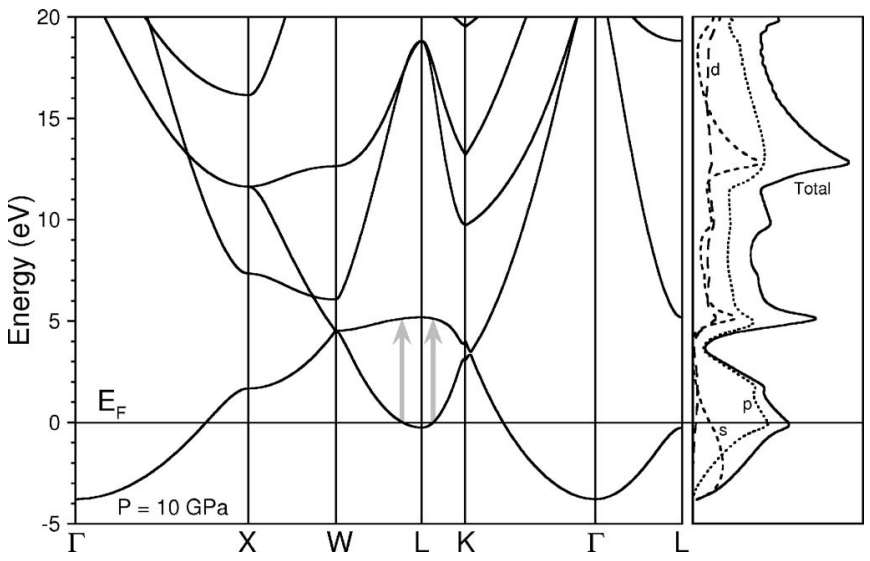

FIG. 2. Calculated energy band structure of fcc lithium at $P=10 \mathrm{GPa}$. Vertical gray arrows show the threshold for optical transitions around $L$. On the right side, both the density of states (DOS) and partial DOS are displayed, where solid, dashed, dotted, and long-dashed lines correspond to total, $s, p$, and $d$ DOSs, respectively.

band structure, dielectric function, and loss function for fcc lithium at $P=40 \mathrm{GPa}$. Comparing Figs. 1 and 3 , it is easy to conclude that the transition threshold $E_{0}$ (due to the energy gap around $L$ ) gradually increases with pressure and induces a blueshift of the main peak in $\operatorname{Im} \epsilon$, increasing the energy corresponding to $B$ plasmon. On the other hand, the energy of $A$ plasmon also increases under pressure due to the increase of the total valence electron density. Observing the evolution of the $B$ plasmon, we see that $\partial \operatorname{Re} \epsilon(\omega) /\left.\partial \omega\right|_{\omega=\omega_{p}^{B}}$ decreases with pressure, which leads to the increase of the relative oscillator strength of this feature over the $A$ plasmon. Additionally, in the 20-30 GPa pressure range the local minimum of $\operatorname{Re} \epsilon$ becomes positive and does not reach zero at energies around $10 \mathrm{eV}$, where the $A$ plasmon is located, leading to an additional broadening of the corresponding peak in the loss function.

Table I summarizes the data on the evolution under pressure of plasmon properties and the threshold energy $E_{0}$ for

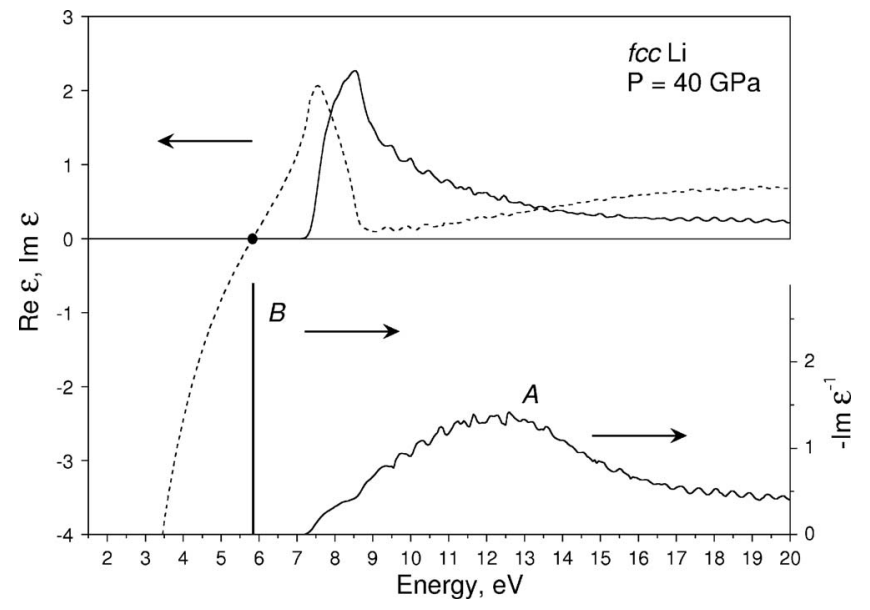

FIG. 3. Calculated dielectric function (upper panel) and loss function (lower panel) for fcc lithium at $P=40 \mathrm{GPa}$. All the notations are the same as in Fig. 1. 


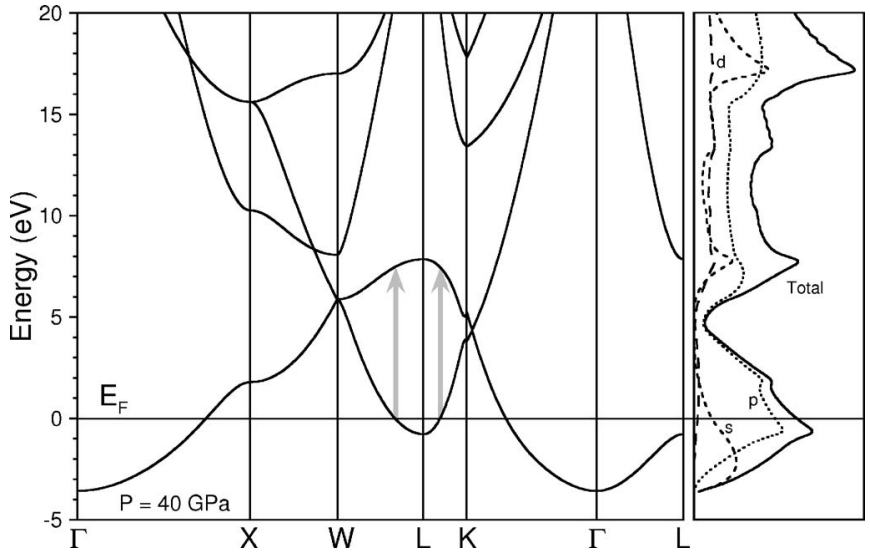

FIG. 4. Calculated band structure of fcc lithium at $P=40 \mathrm{GPa}$. All the notations are the same as in Fig. 2.

optical interband transitions. For completeness, we also present the data for bcc lithium at normal pressure in comparison with other calculations and experiments. It is interesting to note that in bcc lithium at $P=0 \mathrm{GPa}$, the strength of interband matrix elements is not enough to move $\operatorname{Re} \epsilon$ to zero for low energies and momenta, so that only the freeelectron-like plasmon exists.

The existence of $B$ plasmon leads to dramatic modification of optical properties of lithium under pressure. Figure 5 compares the optical constants $(n, \kappa$, and the reflectivity $R$ ) for fcc lithium at 10 and $40 \mathrm{GPa}$. As we can see, when the frequency of incident light is slightly above the $B$ plasmon frequency, it would experience an abrupt decrease in reflectivity with the subsequent total loss of reflectivity at $\sim 5 \mathrm{eV}$ $(\sim 7 \mathrm{eV})$ for $P=10 \mathrm{GPa}(P=40 \mathrm{GPa})$. The cases of $P$ $=20 \mathrm{GPa}$ and $P=30 \mathrm{GPa}$ are similar with vanishing reflectivity at frequencies around 5.9 and $6.5 \mathrm{eV}$, respectively. On

TABLE I. Calculated energies of $A$ and $B$ plasmons in fcc and bcc lithium for different pressures. The width of the $A$ plasmon, $\Delta_{p}^{A}$, and energy gap for optical interband transitions, $E_{0}$, are also shown. For bcc lithium at $P=0$, we compare our result with some other calculated and experimental values.

\begin{tabular}{lccccc}
\hline \hline Pressure & $E_{p}^{A}$ & $\Delta_{p}^{A}$ & $E_{p}^{B}$ & $E_{o}$ & Refs. \\
\hline \multicolumn{5}{c}{$\mathrm{fcc}$} \\
$P=0$ & 7.6 & 2.3 & & 3.3 & \\
$P=10$ & 9.2 & 4.8 & 4.7 & 5.1 & \\
$P=20$ & 10.4 & 5.2 & 5.25 & 6.2 & \\
$P=30$ & 11.5 & 5.4 & 5.6 & 7.0 & \\
$P=40$ & 12.4 & 5.5 & 5.8 & 7.5 & \\
& 7.28 & 2.6 & & & \\
$P=0$ & 7.4 & 2.5 & & & Calc. (Ref. 24) \\
& 7.25 & $\cong 2.9$ & & & Calc. (Ref. 26) \\
& $7.31 \pm 0.06$ & $\cong 2$ & & & Expt. (Ref. 27) \\
& 7.12 & $\cong 2.5$ & & & Expt. (Ref. 28) \\
& $7.08 \pm 0.05$ & $2.2 \pm 0.2$ & & & Expt. (Ref. 29) \\
\hline \hline
\end{tabular}
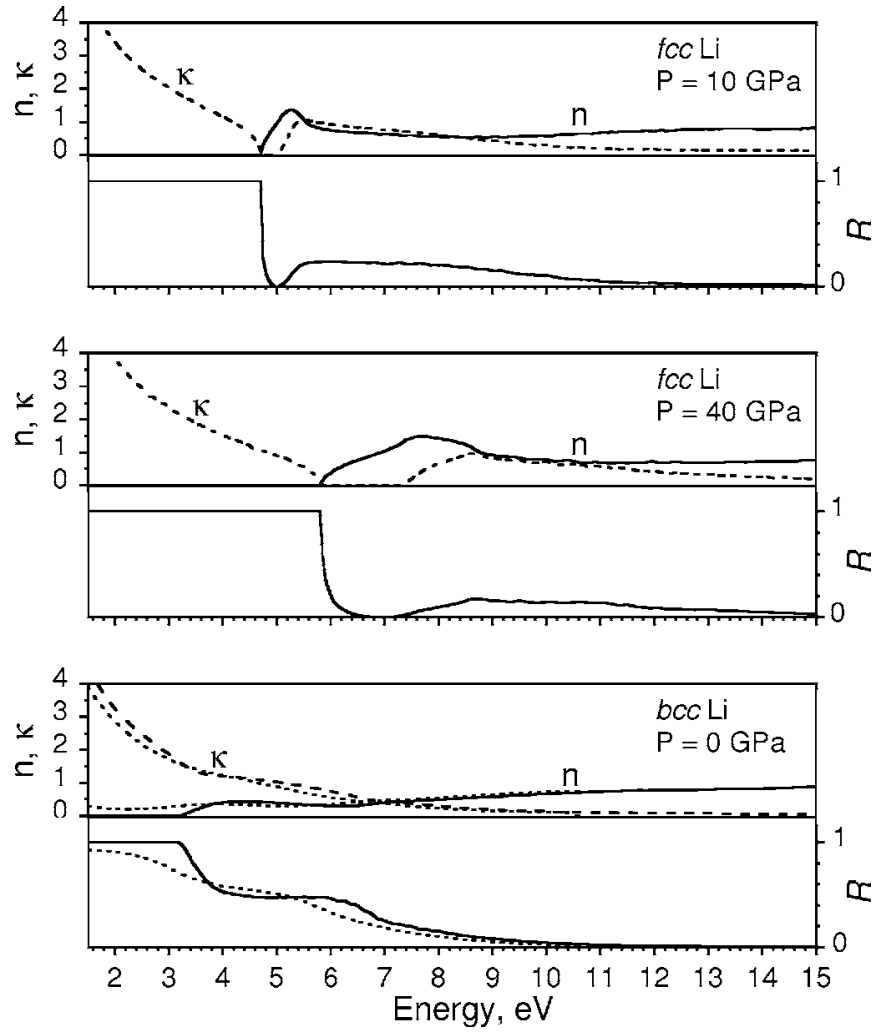

FIG. 5. Refractive indexes $n$ (solid line) and $\kappa$ (dashed line) and reflectivity $R$ for fcc lithium at pressures $P=10 \mathrm{GPa}$ (upper panel) and $P=40 \mathrm{GPa}$ (middle panel) and bcc lithium at $P=0 \mathrm{GPa}$ (lower panel). In the lower panel, experimental data for $n, \kappa$, and $R$ taken from Ref. 30 are shown by dotted lines. Note the remarkable agreement between calculated and measured optical constants for bcc lithium at equilibrium, specially taking into account the difficulties in extraction of experimental data.

the other hand, as can be seen in Fig. 5, reflectivity of fcc lithium is very different from that of bcc lithium.

In conclusion, it is well known that lithium under pressure is far from being a nearly-free-electron-like system, as it is confirmed by the analysis of the evolution of its lowmomentum electronic dynamic response presented here. Electronic localization observed in compressed lithium induces a flattening of electronic bands, so that electronic states around $L$ drop below the Fermi energy under pressure, which allows new optical interband electronic excitations. The sharp increase of $\operatorname{Im} \epsilon$ associated with these excitations makes both the real and the imaginary part of $\epsilon$ go to zero in the lower part of the spectra. Therefore, besides the classical free-electron-like plasmon, a new undamped interband collective mode arises in lithium under pressure, which is expected to induce an abrupt plasma edge in the experimentally observable reflectivity and also affect electronic correlations at low energies. Interestingly, $\mathrm{MgB}_{2}$, which shares with compressed lithium the common feature of having an enhanced superconducting transition temperature, also presents a longlived low-energy interband plasmon. ${ }^{31}$ Now that inelastic $\mathrm{x}$-ray scattering has made possible the measurement of plasmon dispersions under pressure, ${ }^{32}$ it would be interesting to 
test experimentally the presence of the undamped mode found here.

We thank I. A. Nechaev for useful discussions. We acknowledge partial support from the University of the Basque
Country (9/UPV 00206.215-13639/2001), the Basque Unibertsitate eta Ikerketa Saila, the Spanish Ministerio de Ciencia y Tecnología (FIS2004-06490-C0301), and the EC 6th Framework Network of Excellence NANOQUANTA (NMP4-CT-2004-500198).
${ }^{1}$ J. B. Neaton and N. W. Ashcroft, Nature (London) 400, 141 (1999).

${ }^{2}$ A. Bergara, J. B. Neaton, and N. W. Ashcroft, Phys. Rev. B 62, 8494 (2000).

${ }^{3}$ M. Hanfland, K. Syassen, N. E. Christensen, and D. L. Novikov, Nature (London) 408, 174 (2000).

${ }^{4}$ N. E. Christensen and D. L. Novikov, Phys. Rev. Lett. 86, 1861 (2001)

${ }^{5}$ A. Rodriguez-Prieto and A. Bergara, Phys. Rev. B 72, 125406 (2005).

${ }^{6}$ V. E. Fortov et al., JETP Lett. 74, 418 (2001).

${ }^{7}$ K. Shimizu, H. Ishikawa, and K. Amaya, J. Phys.: Condens. Matter 14, 10433 (2002)

${ }^{8}$ K. Shimizu et al., Nature (London) 419, 597 (2002).

${ }^{9}$ V. V. Struzhkin et al., Science 298, 1213 (2002).

${ }^{10}$ S. Deemyad and J. S. Schilling, Phys. Rev. Lett. 91, 167001 (2003).

${ }^{11}$ N. W. Ashcroft, Nature (London) 419, 569 (2002).

${ }^{12}$ K. I. Juntunen and J. T. Tuoriniemi, Phys. Rev. Lett. 93, 157201 (2004).

${ }^{13}$ J. B. Neaton and N. W. Ashcroft, Phys. Rev. Lett. 86, 2830 (2001); M. Hanfland, I. Loa, and K. Syassen, Phys. Rev. B 65, 184109 (2002); M. I. McMahon, S. Rekhi, and R. J. Nelmes, Phys. Rev. Lett. 87, 055501 (2001); M. I. McMahon, R. J. Nelmes, and S. Rekhi, ibid. 87, 255502 (2001); R. J. Nelmes, M. I. McMahon, J. S. Loveday, and S. Rekhi, ibid. 88, 155503 (2002); N. E. Christensen and D. L. Novikov, Solid State Commun. 119, 477 (2001); J. S. Schilling, High Press. Res. 26, 145 (2006).

${ }^{14}$ J. S. Schilling, Treatise on High Temperature Superconductivity (Springer, Hamburg, 2006).

${ }^{15}$ E. Gregoryanz, O. Degtyareva, M. Somayazulu, R. J. Hemley, and H. K. Mao, Phys. Rev. Lett. 94, 185502 (2005).

${ }^{16}$ A. Rodriguez-Prieto, D. Alfe, and A. Bergara (unpublished).

${ }^{17}$ A. Rodriguez-Prieto and A. Bergara, Proceedings of the Joint 20th AIRAPT-43rd EHPRG 2005 Conference (unpublished); arXiv:cond-mat/0505619.

${ }^{18}$ A. Rodriguez-Prieto, A. Bergara, V. M. Silkin, and P. M. Echenique, Phys. Rev. B 74, 172104 (2006); A. Rodriguez-Prieto, A. Bergara, and V. M. Silkin, J. Phys. Soc. Jpn. (to be published).

${ }^{19}$ J. S. Tse, Y. Ma, and H. M. Tununcu, J. Phys.: Condens. Matter 17, S911 (2005); G. Profeta, C. Franchini, N. N. Lathiotakis, A. Floris, A. Sanna, M. A. L. Marques, M. Lüders, S. Massidda,
E. K. U. Gross, and A. Continenza, Phys. Rev. Lett. 96, 047003 (2006); D. Kasinathan, J. Kuneš, A. Lazicki, H. Rosner, C. S. Yoo, R. T. Scalettar, and W. E. Pickett, ibid. 96, 047004 (2006); N. E. Christensen and D. L. Novikov, Phys. Rev. B 73, 224508 (2006).

${ }^{20}$ W. Ku and A. G. Eguiluz, Phys. Rev. Lett. 82, 2350 (1999).

${ }^{21}$ N. Troullier and J. L. Martins, Phys. Rev. B 43, 1993 (1991).

${ }^{22}$ E. Runge and E. K. U. Gross, Phys. Rev. Lett. 52, 997 (1984); M. Petersilka, U. J. Gossmann, and E. K. U. Gross, ibid. 76, 1212 (1996).

${ }^{23}$ The first sum in Eq. (2) was performed with the use of $96 \times 96$ $\times 96 \mathbf{k}$-mesh (442 368 points in BZ), while the second one runs over the occupied and all unoccupied energy bands up to an energy of $50 \mathrm{eV}$ above the Fermi level. We avoid the direct evaluation of $\chi^{0}$ using Eq. (2) by calculating at the first step the spectral function matrix, $S_{\mathbf{G}, \mathbf{G}^{\prime}}(\mathbf{q}, \omega)$, from which the imaginary part of the $\chi_{\mathbf{G}, \mathbf{G}^{\prime}}^{0}(\mathbf{q}, \omega)$ is readily obtained (Ref. 33). Subsequently, the real part of $\chi^{0}$ is obtained from $\operatorname{Im} \chi^{0}$ through the Hilbert transform. Numerically, in the evaluation of $S_{\mathbf{G}, \mathbf{G}^{\prime}}(\mathbf{q}, \omega)$ the $\delta$ function was replaced by a Gaussian with a broadening parameter of $0.1 \mathrm{eV}$ (Ref. 34).

${ }^{24}$ K. Karlsson and F. Aryasetiawan, Phys. Rev. B 52, 4823 (1995).

${ }^{25}$ R. E. Alonso, S. Sharma, C. Ambrosch-Draxl, C. O. Rodriguez, and N. E. Christensen, Phys. Rev. B 73, 064101 (2006).

${ }^{26}$ Y.-G. Jin and K. J. Chang, Phys. Rev. B 59, 14841 (1999).

${ }^{27}$ J. P. Hill, C.-C. Kao, W. A. C. Caliebe, D. Gibbs, and J. B. Hastings, Phys. Rev. Lett. 77, 3665 (1996).

${ }^{28}$ C. Kunz, Z. Phys. 196, 311 (1966).

${ }^{29}$ T. Kloos, Z. Phys. 265, 225 (1973).

${ }^{30}$ D. W. Lynch and W. R. Hunter, in Handbook of Optical Constants of Solids II, edited by E. D. Palik (Academic Press, San Diego, 1991).

${ }^{31}$ V. P. Zhukov, V. M. Silkin, E. V. Chulkov, and P. M. Echenique, Phys. Rev. B 64, 180507(R) (2001); W. Ku, W. E. Pickett, R. T. Scalettar, and A. G. Eguiluz, Phys. Rev. Lett. 88, 057001 (2002); S. Galambosi, J. A. Soininen, A. Mattila, S. Huotari, S. Manninen, G. Vankó, N. D. Zhigadlo, J. Karpinski, and K. Hämäläinen, Phys. Rev. B 71, 060504(R) (2005); Y. Q. Cai et al., Phys. Rev. Lett. 97, 176402 (2006).

${ }^{32}$ I. Loa et al., Proceedings of the Joint 20th AIRAPT-43rd EHPRG 2005 Conference (unpublished).

${ }^{33}$ F. Aryasetiawan and O. Gunnarsson, Phys. Rev. B 49, 16214 (1994).

${ }^{34}$ T. Miyake and F. Aryasetiawan, Phys. Rev. B 61, 7172 (2000). 